\title{
Mediaciones Tecnológicas para el desarrollo de la competencia semántica comunicativa- lectura inferencial en básica primaria.
}

Technological Mediations for the development of communicative semantic competition inferential reading in primary basics.

\author{
María C. Bárcenas Morales \\ Universidad de Sucre - Colombia \\ mariabarcenasmorales@gmail.com \\ http://orcid.org/0000-0003-3248-7303 \\ Paolo D. Mercado Gómez \\ Universidad de Sucre - Colombia \\ padamego@gmail.com \\ http://orcid.org/0000-0001-8428-8301 \\ Nohemy Carrascal Torres. \\ Universidad de Córdoba - Colombia \\ ncarrascal@gmail.com \\ https://orcid.org/0000-0003-4925-0935
}




\section{Resumen}

El presente artículo describe la metodología, resultados y conclusiones derivadas del estudio "Mediaciones Tecnológicas para el desarrollo de la Competencia Semántica Comunicativa- lectura Inferencial en estudiantes de Básica Primaria" desarrollado en la Institución Educativa José María Berástegui, municipio de Ciénaga de Oro, Departamento de Córdoba, con el propósito de potenciar el desarrollo de la competencia semántica comunicativa de la lectura inferencial en estudiantes de tercer grado de básica primaria. A partir de la reflexión de estudiantes, docentes y padres de familia se diseñan ambientes mediados con TIC fundamentados conceptualmente en el Enfoque Semántico Comunicativo.

El proceso investigativo se desarrolla en cuatro fases: una fase diagnóstica la cual se estructura a partir del acompañamientos en el aula, evaluación diagnóstica, revisión y reflexión sobre los aspectos registrados en el observador del alumno; los datos obtenidos permiten construir colectivamente un plan de acción, seguidamente se desarrollan las acciones planeadas y se finaliza con la fase de evaluación reflexión sobre el proceso desarrollado, las transformaciones obtenidas en las prácticas de aula y la potenciación en los estudiantes de grado tercero de la básica primaria en lectura inferencial.

Conceptualmente se fundamenta en la teoría de Cassany (2006), la cual señala que "Leer es recuperar el valor semántico de cada palabra y relacionarlo con el de las palabras anteriores y posteriores", en este mismo sentido Van Dijk (1999), establece que la lectura está mediatizada por la memoria social, elementos esenciales en el proceso lector. Los resultados evidencian cambios en la actitud, motivación y participación en las actividades diseñadas a través de TIC, mejorando la comprensión lectora a nivel inferencial. Este diseño de nuevos ambientes de aprendizaje mediados con TIC para la enseñanza de la lectura favorece el aprendizaje cooperativo, colaborativo, el reconocimiento y valoración de los saberes de los estudiantes y el enriquecimiento de los contextos de aula.

\section{Abstract}

This article describes the methodology, results and conclusions derived from the study "Technological Mediations for the Development of Communicative Semantic Competence - Inferential Reading in Primary School Students" developed in the Educational Institution José María Berástegui, municipality of Ciénaga de Oro, Department of Córdoba, with the purpose of promoting the development of communicative semantic competence in inferential reading in third grade primary school students. From the reflection of students, teachers and parents, environments are designed mediated with information and communication technologies (TIC) conceptually based on the Semantic Communicative Approach.

The research process is developed in four phases: a diagnostic phase which is structured from the accompaniments in the classroom, diagnostic evaluation, review and reflection on the aspects recorded in the student's observer; the data obtained allow for the collective construction of an action plan, followed by the development of planned actions and ends with the phase of reflection on the 
process developed, the transformations obtained in classroom practices and the empowerment of third grade students in primary basic inferential reading.

Conceptually, it is based on the theory of Cassany (2006), which points out that "Reading is recovering the semantic value of each word and relating it to that of the previous and subsequent words", in this same sense Van Dijk (1999) establishes that reading is mediated by social memory, essential elements in the reading process. The results show changes in attitude, motivation and participation in activities designed through information and communication technologies (TIC), improving reading comprehension at an inferential level. This design of new learning environments mediated with information and communication technologies (TIC) for the teaching of reading favours cooperative and collaborative learning, the recognition and valuation of students' knowledge and the enrichment of classroom contexts.

\section{Palabras Clave}

Lectura Inferencial, Comprensión Lectora, Habilidades Comunicativas, Estrategias de Enseñanza y Aprendizaje, Enfoque Semántico Comunicativo.

\section{Keywords}

Inferential Reading, Reading Comprehension, Communicative Skills, Teaching and Learning Strategies, Communicative Semantic Approach.

\section{Introducción}

Atendiendo a la concepción de la educación propuesta por el Ministerio de Educación Nacional (MEN), centrada en la noción de competencia comunicativa planteada por Dell Hymes (1972), introduce una visión más pragmática del lenguaje, resaltando algunos aspectos socio-culturales que resultan determinantes en cada acto comunicativo, el cual permite desde edades primarias desarrollar la competencia comunicativa, proporcionando de manera integrada actitudes, valores, motivaciones y todo lo que tiene que ver con la lengua. A partir de estos planteamientos se deriva el denominado enfoque semántico comunicativo, que atiende a la construcción del significado y el sentido de tomar el acto de comunicación e interacción como unidad de trabajo, atendiendo a la construcción del significado de un texto (Usuga, 2010), partiendo de la base, que la inferencia como proceso, es un elemento relevante en la construcción de la comunicación (Parodi, 2005) y es considerada como una operación mental, ejecutada normalmente por el ser humano (Cassany et al., 2000).

Particularmente, el propósito del presente estudio fue potenciar el desarrollo de la competencia semántica comunicativa- lectura inferencial en estudiantes de tercer grado de básica primaria de la institución educativa José María Berástegui, a través del diseño e implementación de ambientes mediados con TIC, debido a 
que por medio de las prácticas de aula y seguimientos trimestrales realizados por los docentes, se observó un alto índice de dificultad, en la que alrededor del 70\% de los estudiantes de $3^{\circ}$ grado de básica primaria, presentaban problemas a la hora de comprender textos escritos, evidenciado con las siguientes acciones: Dificultad al relacionar personajes y acontecimientos que se desarrollan en el espacio temporal de un texto, confusión en el significado de las palabras u omitían aquellas que le daban sentido al texto, el uso del vocabulario a la hora del expresarse oralmente no era el adecuado, de igual manera se percibía la desmotivación permanentemente para leer y escribir, mostrando apatía y desinterés por participar en las distintas actividades correspondientes a estas habilidades comunicativas, cuyos efectos se evidenciaron en el rendimiento escolar, teniendo en cuenta que la lectura es básica para los futuros aprendizajes.

Para ello fue necesario, identificar los niveles de desarrollo de la competencia semántica comunicativa-lectura inferencial en los estudiantes, haciendo la respectiva caracterización a las estrategias pedagógicas utilizadas por los docentes para la enseñanza de la lectura y de esta manera se inició un proceso de diseño e implementación de ambientes propicios para el desarrollo de la competencia, usando las herramientas TIC, en este mismo sentido, Marqués (2000) señala que las TIC, permiten un más rápido aprendizaje en mejor tiempo por medio de los diversos recursos educativos que utiliza, los cuales hacen parte de las herramientas con que cuentan los niños y jóvenes en la cotidianidad que hoy viven.

De igual forma, se evaluaron los niveles de desarrollo de la competencia semántica comunicativa-lectura inferencial, mediadas por las herramientas TIC, haciendo uso de la (IAP), caracterizada por ser una metodología que apunta a la producción de un conocimiento propositivo y trasformador, mediante un proceso de debate, reflexión colectiva de saberes entre los diferentes actores de un territorio con el fin de lograr la transformación social (Bárcenas \& Mercado 2017), atendiendo las etapas o fases, explicadas por Kemmis \& Mc Taggart, (1988), las cuales permiten el mejoramiento de la práctica, la comprensión y la mejora de la situación en la que tiene lugar la práctica por medio de IV fases las cuales son: FASE I. Diagnóstico, en el que se realiza una reflexión inicial por medio de una prueba evaluativa; FASE II, Diseño del plan de acción que constituye la determinación, selección y diseño de estrategias; FASE III, Implementación de la acción en el que se aplican las estrategias diseñadas de acuerdo a lo observado y FASE IV, la evaluación mediante la cual se reflexiona acerca del proceso llevado a cabo. 


\section{Referentes teóricos}

La lectura es uno de los aprendizajes más importantes, indiscutidos e indiscutibles, que proporciona la escolarización, donde la alfabetización es la puerta de entrada a la cultura escrita y a todo lo que ella comporta: una cierta e importante socialización, construcción de conocimiento y acceso a todo tipo de información. Además, implica en el sujeto capacidades cognitivas superiores. Quien aprende a leer eficientemente desarrolla, accede y participa a las producciones culturales y científicas. En definitiva, la lectura se convierte en un aprendizaje transcendental para la escolarización y para el crecimiento intelectual de la persona Cassany (2001); por lo que este proceso requiere del dinamismo y entusiasmo de todo aquel que hace el papel de orientador, dentro del aula de clases.

Con respecto a la significación Cassany (2006) sostiene que leer es recuperar el valor semántico de cada palabra y relacionarlo con el de las palabras anteriores y posteriores. El contenido surge de la suma del significado de todos los vocablos y oraciones, permitiendo de esta manera valorar los conocimientos previos que tiene el estudiante, conocimientos valiosos que permiten crear nuevas ideas, creando la posibilidad de nuevos aprendizajes. En este mismo sentido, Solé (1992) resalta la importancia de la comprensión lectora puesto que la lectura "es significativa para las personas", y plantea además que esto solo puede hacerlo mediante una lectura individual, y que la misma, le permita detenerse, pensar, recapitular, relacionar la información nueva con el conocimiento previo que posee, es así, donde estos, no se pueden pasar por alto, debido a que fortalecen el aprendizaje, haciendo de este más significativo.

Ahora bien, dentro de las distintas manifestaciones de la actividad lingüística, sean de naturaleza verbal o no verbal, se dan dos procesos: la producción y la comprensión. La producción hace referencia al proceso por medio del cual el individuo genera significado, lo que se toma desde el ámbito contextual, enlazado con sus vivencias, ya sea con el fin de expresar su mundo interior, transmitir información o interactuar con los otros. Entre tanto, la comprensión tiene que ver con la búsqueda y reconstrucción del significado y sentido que implica cualquier manifestación lingüística. Estos dos procesos -comprensión y producciónsuponen la presencia de actividades cognitivas básicas como la abstracción, el análisis, la síntesis, la inferencia, la inducción, la deducción, la comparación, la asociación, MEN (2006).

Teniendo en cuenta todo lo anterior, es importante resaltar algunos niveles de pensamiento de la información y estrategias para el desarrollo del pensamiento crítico de Maureen Priestley (1996), donde se evidencian la progresión de los niveles de lectura y las técnicas y estrategias para desarrollar el pensamiento crítico del ser humano (ilustración 1). 


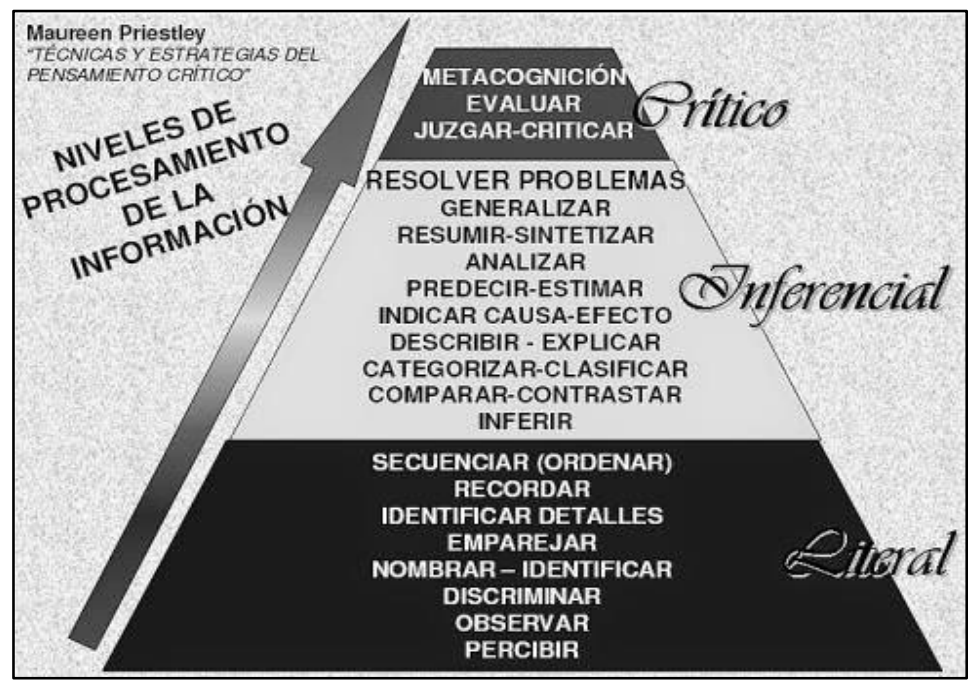

Fuente: Priestley (1996)

Este estudió centró su interés particularmente en el nivel inferencial, el cual también es definido por el MEN(1998), como la capacidad para deducir y concluir acerca de aquellos componentes del texto que aparecen implícitos; en su efecto, esta estructura de los niveles de procesamiento de la información, brinda la oportunidad de saber que se debe tener en cuenta en cada uno de los niveles de procesamiento de la información, iniciando desde una base literal, avanzando progresivamente a desarrollar la capacidad de inferir, hasta llegar al nivel crítico.

A partir de lo anterior, se debe replantear el rol del docente hacia el uso de nuevas metodologías de trabajo (Marín \& Armentia, 2009), donde el maestro sea orientador que direcciones y facilite el uso de las mediaciones tecnológicas, para cambiar los escenarios de aprendizaje. Díaz (2005), apunta que son necesarias 'nuevas formas de cotidianidad', para conformar nuevas miradas al campo de la tecnología apropiada y crítica (Fainholc, 2000) que permitan reconfiguran las prácticas en tecnología educativa.

Por otra parte, los estudios desarrollados por Gil (2011), Madero (2011), Villalonga et al. (2014), y Vázquez (2016) permiten afirmar que el ambiente en que se encuentra el estudiante representa un factor influyente en el desarrollo de habilidades de la comprensión lectora, también concurren en la necesidad de empezar el proceso desde temprana edad en diferentes situaciones, de tal forma que se involucre al niño en la búsqueda de información implícita en un texto (ilustración, noticias, esquemas entre otros), debido a que este, cuando empieza la actividad lectora tiene las herramientas necesarias para relacionar el contenido del texto con el contexto que lo rodea. De manera que los ambientes enriquecidos con tecnología permiten formular estrategias para que el estudiante participe activamente y reflexione sobre su proceso de comprensión lectora, en el cual tiene la oportunidad de valorar, autorregularse. 


\section{Metodología}

Este estudio se define desde un enfoque cualitativo, con una metodología de tipo investigación, acción, participación (IAP), la cual se caracteriza por favorecer la producción participativa de conocimiento propositivo y trasformador, mediante un proceso de debate, reflexión colectiva de saberes entre los diferentes actores de un territorio con el fin de lograr la transformación social.

Ilustración 2. Fases de la investigación.

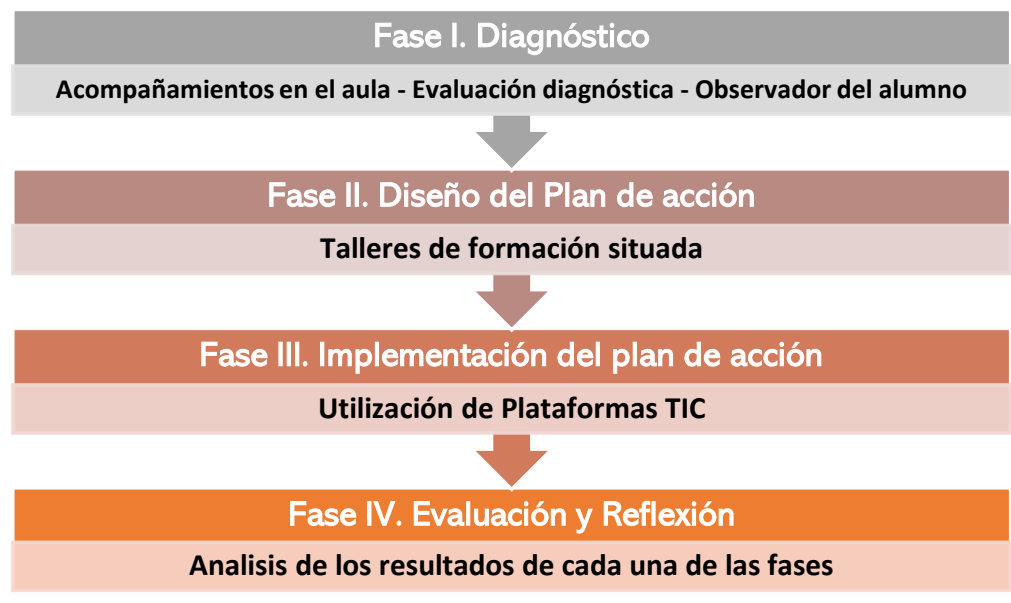

Fuente. Adaptación a partir de Kemmis \& Mc Taggart, (1988).

En la ilustración 2, se aprecian las etapas que se llevaron a cabo en el proceso de investigación, para lo cual se tuvo en cuenta lo propuesto por Kemmis \& Mc Taggart, (1988):

\section{Fase I. Diagnóstico}

En esta fase se identificaron las características de la población objeto de estudio con el fin de diagnosticar las debilidades que tienen los estudiantes y la docente, a través de las siguientes técnicas:

Acompañamientos en el aula: Mediante un proceso de observación reflexión, utilizando un protocolo se evidencia el desarrollo de las clases, empleando las siguientes etapas: (a) reflexión sobre la planeación de clase. En esta etapa se reportan el material a utilizar, implementación de las actividades de aprendizaje, el uso efectivo del tiempo y forma de evaluación del docente. (b) Visita en el aula: se revisa la forma de expresión y uso adecuado del vocabulario del docente. (c) Preparación para la realimentación: se clasifica la información recopilada de las anteriores etapas, según el clima, gestión de aula, didáctica y evaluación formativa. (d) Realimentación: se organiza un grupo de discusión, entre docente e investigadores para debatir sobre la problemática establecida en el primer seguimiento, estableciendo acuerdos para posibilitar mejoras en las estrategias pedagógicas en las clases. 
Evaluación diagnóstica: se realizó para determinar el nivel de lectura inferencial en que se encontraban los estudiantes a través de una prueba diagnóstica (tipo ICFES), conformada por preguntas inferenciales de problema o solución, dos de objeto, dos de tiempo, cinco de causa y efecto, cuatro de actitud, una de categoría, dos de agente, una de acción, una de lógica y una de lugar. Para identificar la confiabilidad del instrumento (Hernández, 2016), se aplicó la prueba de alfa de Cronbach con el software SPSS (Startical Product and Service Solutions Version 24).

Observador del alumno: Se realiza revisión documental, utilizando la bitácora de registro en la cual se establecen los posibles avances, deficiencias y habilidades durante la implementación de la fase anterior. Consta de una primera parte donde se registran los datos básicos del estudiante, nombre de los padres, ocupación, teléfono, entre otros. En la segunda parte se emplea un formulario compuesto por el desempeño de los estudiantes, donde se describen las debilidades y/o fortalezas encontradas en los mismos. Finalmente, se contrastan las estrategias del docente y los resultados de los desempeños del estudiante, a través de una triangulación (Patton, 2002) de verificación y comparación de la información de anteriores etapas.

\section{Fase II. Diseño del Plan de acción}

Con base en los resultados obtenidos en la primera fase, se diseña en conjunto con el docente talleres para resolver las problemáticas identificada, utilizando mediaciones tecnológicas en la enseñanza de la lectura inferencial y sus diferentes tipos (inferencias de tiempo, de lugar, de problema, de causa y efecto, de agente, entre otras). Los talleres se desarrollan a través de plataformas virtuales, videos y lecturas compartidas, donde los estudiantes puedan realizar inferencias a partir de la comprensión lectora de las mismas.

\section{Fase III. Implementación del plan de acción}

En esta fase se desarrolló el plan de acción, a través de talleres ejecutados en las dos plataformas virtuales: plataforma virtual Offline "Aprendamos a inferir" y Educaplay. Los talleres contienen, imágenes, animaciones y videos enfocados a mejorar la competencia semántica comunicativa específicamente lectura inferencial.

\section{Fase IV. Evaluación y Reflexión}

En esta fase se hizo una revisión de los resultados obtenidos de las acciones implementadas en cada una de las etapas anteriores. La información obtenida durante todo el proceso se analiza y se identifican los logros alcanzados y los aspectos que se deben mejorar, realizando su valoración de acuerdo a la estrategia utilizada. En la evaluación de este proceso, se miran los propósitos 
alcanzados y los no alcanzados, lo que le va a permitir a los estudiantes, reflexionar acerca de la incidencia de esta investigación en el desarrollo de la competencia semántica comunicativa específicamente la lectura inferencial, así como también le permite a la docente discurrir sobre su práctica pedagógica, su desarrollo a nivel personal y su desempeño profesional.

\section{Participantes}

Para la realización de esta investigación participaron 22 estudiantes del grado tercero, 9 niños y 13 niñas, los cuales provienen de familias campesinas, con edades entre los 8 y 11 años de edad. Además se contó con el apoyo y colaboración del docente director de grupo de este grado. Se aplicó un muestreo de tipo intencional opinático, en el cual los elementos del universo que entran en la muestra, según Guerrero et al. (1986) se escogen conforme a una norma o guía prevista y deliberadamente establecida; de igual forma se escogió este grupo, dado el acercamiento, la oportunidad y accesibilidad a la Institución Educativa José María Berástegui.

\section{Resultados y Discusión}

Los resultados se presentan de acuerdo con las fases de la intervención metodológica, las cuales permitieron determinar soluciones factibles y participativas a los problemas referentes al desarrollo de la competencia semántica comunicativa, específicamente lectura inferencial en los estudiantes.

Fase I. Diagnóstico.

En esta fase se identificaron las características de la población objeto de estudio: Problemas, expectativas y reflexión sobre el desarrollo de la competencia semántica comunicativa en el nivel de lectura inferencial, que corresponde con el primer propósito específico de esta investigación.

En este orden de ideas, se iniciaron las conversaciones con la docente del grado tercero de básica primaria sobre los desempeños en las pruebas saber de los años 2015 y 2016, así como aspectos críticos reflejados en los desempeños de los estudiantes en el momento de realizar compresiones de lecturas tales como, dificultad al relacionar los personajes y acontecimientos que se desarrollan en el espacio temporal de un texto, confusión en el significado de las palabras, puesto que presentan omisión cambiando el sentido al texto, el uso del vocabulario a la hora del expresarse de manera oral no es el adecuado y se observa una desmotivación permanente a la hora de leer, mostrando apatía y desinterés por participar en las distintas actividades correspondientes a este proceso de lectura.

De igual manera, se hizo un seguimiento a la docente, por medio de dos acompañamientos en el aula, para mirar los aspectos relacionados con el desempeño pedagógico y el uso de herramientas propias para facilitar el 
aprendizaje en los estudiantes, mirar la actitud de esta frente a su quehacer pedagógico y reflexionar acerca de la metodología utilizada en el aula para el desarrollo de sus clases, es así, como por medio de una rúbrica de acompañamiento en el aula, suministrado por el programa PTA, se procedió a realizar el acompañamiento descrito de la siguiente manera.

\section{Acompañamiento en el aula.}

Tabla 1. Descripción de la etapa de acompañamientos en el aula.

\begin{tabular}{|c|c|}
\hline \multicolumn{2}{|r|}{ Etapa de Acompañamiento en el aula } \\
\hline Propósitos & $\begin{array}{l}\text { Determinar las prácticas metodológicas y evaluativas de la docente, desde la } \\
\text { reflexión individual y colectiva. }\end{array}$ \\
\hline $\begin{array}{l}\text { Reflexión sobre } \\
\text { la planeación } \\
\text { de clase }\end{array}$ & $\begin{array}{l}\text { En esta fase se entró a dialogar con la docente, para identificar los objetivos a } \\
\text { desarrollar en el aula de clase con los estudiantes, verificando de esta manera, el } \\
\text { uso de los materiales didácticos a utilizar para el desarrollo de las actividades } \\
\text { planeadas. }\end{array}$ \\
\hline $\begin{array}{l}\text { Visita en el } \\
\text { aula }\end{array}$ & $\begin{array}{l}\text { En esta fase se analizaron los materiales y evidencias que utilizó la docente en los } \\
\text { siguientes aspectos: La interacción entre estudiantes está orientada por el buen } \\
\text { trato y el respeto, el docente crea un ambiente de aprendizaje poco agradable y } \\
\text { accesible, sin considerar la organización del espacio físico y los recursos } \\
\text { disponibles, lo que no permite la participación activa en los estudiantes. } \\
\text { Los materiales usados en esta actividad fueron: libros, textos de lectura y de } \\
\text { consulta, lo que pudimos analizar, que gran parte de la desmotivación de los } \\
\text { estudiantes, se debía a la selección de libros y lecturas muy antiguas y } \\
\text { desactualizadas, donde no despertaban ningún interés en los estudiantes, por } \\
\text { consiguiente, las actividades no alcanzarían a lograr el impacto en el aprendizaje de } \\
\text { los niños. } \\
\text { Con respecto al clima de aula, se percibió un docente tradicionalista, donde daba } \\
\text { solo órdenes y entregaba el material y no permitía alguna intervención por parte de } \\
\text { los estudiantes, el material impreso y la lectura a trabajar no fueron motivadores, lo } \\
\text { que logró solo llamar la atención de la mitad del curso. } \\
\text { Se pudo observar que en lo que tiene que ver con la gestión del aula, la docente } \\
\text { manejó un lenguaje claro, sin enfatizar en las estrategias de participación de los } \\
\text { estudiantes; al colocarlos en parejas para realizar la actividad, se evidenció } \\
\text { desorden y poca atención por parte de los estudiantes cabe anotar que la } \\
\text { interacción entre la docente y los se manejó con buen trato lo que ayudo en algunos } \\
\text { educandos asimilar en pequeñas proporciones las lecturas inferenciales que } \\
\text { estaban realizando. }\end{array}$ \\
\hline $\begin{array}{l}\text { Preparación } \\
\text { para la } \\
\text { realimentación }\end{array}$ & $\begin{array}{l}\text { Clima de aula: Se sugirió que la interacción entre el docente y sus estudiantes, } \\
\text { siempre este orientada por el buen trato, la clase se debe dar con normas claras, } \\
\text { conocidas y que todos puedan seguirlas, } \\
\text { Gestión de aula: El docente debe dar instrucciones claras para el desarrollo de las } \\
\text { actividades, así como los estudiantes cuentan con tiempo necesario para } \\
\text { desarrollar las actividades solicitadas y desarrollar el proceso de aprendizaje. } \\
\text { Enseñanza aprendizaje: Se deben involucrar a todos los estudiantes, cognoscitiva y } \\
\text { activamente en actividades planeadas y orientadas al aprendizaje, a través de la } \\
\text { interacción entre ellos, preguntas, respuestas, acciones, reacciones, propuestas y } \\
\text { creaciones. } \\
\text { Evaluación formativa: Lograr que los estudiantes participen del uso de diferentes }\end{array}$ \\
\hline
\end{tabular}




\section{Evaluación diagnóstica.}

En esta fase se analizaron los resultados obtenidos de la aplicación del instrumento, en los estudiantes, para evidenciar las dificultades que se presentaron en los procesos de comprensión lectora, específicamente en el nivel inferencial.

El método de Investigación Acción - Participación que se utilizó en esta investigación, permitió al docente profundizar los elementos críticos que problematizan sus acciones pedagógicas y evaluativas para mejoramiento en su rol de orientador, identificando las debilidades en su proceso educativo, analizando los resultados obtenidos por los estudiantes en la prueba diagnóstica y caracterizándolos según el nivel de desempeño en las competencias semántica comunicativa desde el nivel inferencial, los resultados coinciden con la forma como el docente desarrolla la clase al ser esta reproductiva y repetitiva. En este sentido, esta investigación es una oportunidad de aporte al proceso educativo, porque permitió la transformación de la práctica pedagógica y evaluativa desde la iniciativa de reflexión y análisis, donde el mismo docente intervino en los cambios voluntariamente. Los estudiantes mostraron un nivel de motivación muy bajo, puesto que se sienten apáticos en realizar las actividades señaladas por la docente frente a la lectura inferencial, las estrategias pedagógicas utilizadas por la docente que deslumbran en los resultados obtenidos en la prueba diagnóstica y en los resultados de las pruebas saber en el grado tercero.

Esta actividad inicial fue el fundamento para el desarrollo del primer propósito de la investigación, precisando información por parte de los estudiantes, docentes y padres de familia, se observa la necesidad de hacer revisión de la misma, enfocando estrategias que fomenten la superación personal desde el proceso educativo; para ello fue necesaria la identificación de las características de los estudiantes en cuanto a su motivación, intereses, autoestima y su visión a futuro, los factores que inciden en ello y el diseño de estrategias que permitan introducir mejoras.

\section{Observador del estudiante.}

Con este instrumento se encontraron las áreas donde pueden mejorar los estudiantes según las dificultades del tipo de inferencia. Este instrumento permitió la comunicación entre la triada el docente, estudiante y padre de familia, con el fin de dar a conocer el estado actual de los educandos y como estos pueden mejorar la calidad de educación desde casa.

\section{Fase II. Diseño del plan de Acción.}

En esta fase se involucraron todos los participantes de la investigación, estudiantes y docentes, en los que se analizaron los aspectos relacionados con la competencia semántica comunicativa, para darle cumplimiento al segundo propósito que hacía referencia a la caracterización de las estrategias pedagógicas que utilizó la docente para la enseñanza de la lectura en el grado tercero, de esta manera, se realizó una socialización del diagnóstico elaborado 
en la fase anterior, en la perspectiva del desarrollo de, motivación y los diferentes tipos de inferencias, así como de cada una de las situaciones presentadas en el aula concernientes al proceso de enseñanza que involucra directamente al docente y las derivaciones de las observaciones y diálogos con padres de familia. Analizando en forma activa y participativa los factores que influyen en ello.

En esta segunda etapa de la investigación se diseñó un plan de acción que surgió de los encuentros entre estudiantes y docentes, en virtud del análisis del diagnóstico inicial descrito anteriormente. Para concretar el plan, se realizaron diferentes reuniones durante el mes de Febrero y Marzo del año 2017, las cuales permitieron establecer las nuevas estrategias de intervención.

De acuerdo al análisis consensuado entre estudiantes y docentes, se abordaron temáticas y se plantearon alternativas de solución al problema descrito, además se reflexionó sobre las fortalezas y debilidades que poseen a nivel institucional y personal, como elementos fundamentales para llevar a cabo las acciones de mejoramiento.

Ilustración 3. Fortalezas y debilidades personales e institucionales
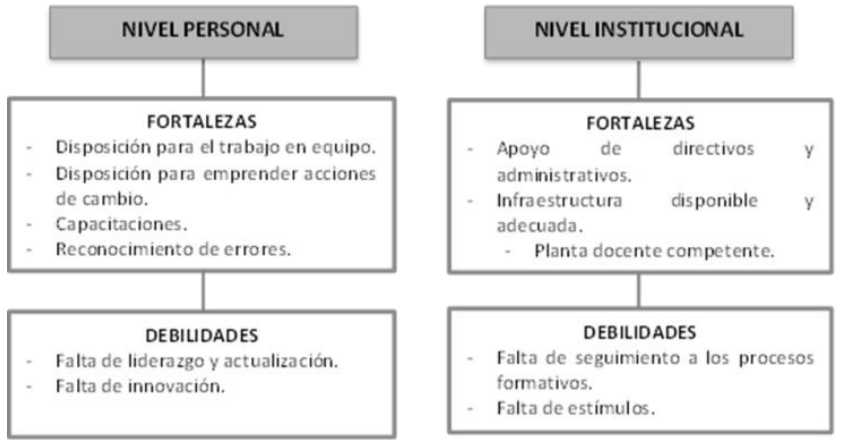

Fuente: Adaptación a partir de Jomabe (2017)

\section{Fase III. Implementación del plan de acción.}

En esta fase se desarrolló el plan de acción elaborado en forma conjunta por los sujetos de investigación, para darle viabilidad al tercer propósito de la investigación como lo era el diseño e implementación de ambientes mediados con TIC en la enseñanza de la lectura inferencial en grado tercero de la institución educativa José María Berástegui. A partir del proceso de observación detallada y análisis de la información obtenida, se identificaron y reconocieron los aspectos críticos y procesos llevados a cabo en relación a la superación personal desde la educabilidad y la las diversas formas de enseñar y se definieron procedimientos y estrategias y a modo de propuesta acorde a la problemática encontrada, es así, como se plantearon talleres en los que se realizarían conceptualizaciones, socializaciones, informes, análisis y reflexiones acerca de la temática lo cual permitió reconocer la situación de los estudiantes de la institución y la práctica de la docente, que se ha venido dando y de acuerdo con esto, mejorar la práctica 
evaluativa con el fin de tener un mejor empoderamiento del conocimiento desde lo cotidiano.

La implementación de la mediación tecnológica fue una estrategia muy valiosa que le brindó a los docentes la posibilidad de mejorar sus prácticas de aula, crear entornos de aprendizajes más dinámicos e interactivos para complementar el proceso de enseñanza y aprendizaje de sus estudiantes, facilitar el trabajo en equipo y el cultivo de actitudes sociales con la Comunidad de Aprendizaje, de igual manera, tuvo un impacto en los alumnos, les ayudó a desarrollar la creatividad e imaginación frente a diferentes situaciones, mostraron una actitud más participativa y con más motivación, mostrando autonomía en las diversas actividades académicas, logrando mejorar notoriamente en la comprensión lectora, específicamente en el nivel inferencial y en las diversas áreas a nivel transversal, ya que los recursos multimedia, páginas web y otros medios tecnológicos le brindaron la posibilidad de mejorar el proceso enseñanza aprendizaje y ver las clases desde otra perspectiva.

Según Somekh, B (2007) citado por Sáez (2011), las TIC contribuyen al aprendizaje autónomo, pues los estudiante con la orientación del docente, identifican con mayor eficiencia y efectividad la información que necesita por medio de fuentes diversas, alcanzando resultados sin sentirse presionado, lo cual fortalece los procesos cognitivos y genera ambientes propicios de aprendizaje para que los estudiantes desarrollen nuevas habilidades lectoras y comunicativas. Adicionalmente, Sepúlveda y Calderón (2007) consideran que el proceso de enseñanza aprendizaje se ha enriquecido por la integración de las TIC ya que para los estudiantes herramientas como el computador producen estímulos placenteros, la atención, la necesidad que amplían y mejoran la enseñanza tradicional.

\section{Fase IV. Evaluación y Reflexión.}

Este punto se analizó desde una perspectiva cualitativa y cuantitativa para demostrar desde un análisis estadístico la validez del avance en el desempeño de la competencia. Una vez realizada la intervención por medio de las mediaciones tecnológicas, se procedió a evaluar los niveles de desarrollo de la competencia semántica comunicativa-lectura inferencial, por medio de la aplicación de una prueba evaluativa, para mirar el impacto e intervención de las herramientas TIC, frente a las diferentes claves en el nivel inferencial, para el cual después de ser aplicada, se hizo el respectivo análisis donde se obtuvo la siguiente información:

En el tipo de inferencia de problemas de solución, los porcentajes estuvieron con una mejoría del $20 \%$ más que en las fase del diagnóstico arrojando un resultado de $72,7 \%$ mejorando considerablemente por el uso de las mediaciones tecnológicas, así mismo, el grupo muestra una comprensión en este tipo de preguntas y la estructura que se articula en torno a la presentación de uno o varios problemas, posteriormente al planteamiento de sus posibles soluciones.

Como apoyo pedagógico las TIC no se limitan a emitir información; influyen en procesos de enseñanza-aprendizaje. De acuerdo con Marqués (2001) citado por Sáez (2011), las ventajas de las TIC en la enseñanza de la lectura son: la 
interacción que permite imágenes, videos (imágenes en movimiento), música y otros textos simultáneamente; la continua actividad intelectual y el desarrollo de la iniciativa, así como la alfabetización digital y audiovisual, el alto grado de interdisciplinariedad, pues distintos formatos están a la mano al mismo tiempo, desarrollo de habilidades de búsqueda y selección de información, mejora de las competencias de expresión y creatividad, fácil acceso a mucha información de todo tipo, visualización de simulaciones, mayor comunicación entre profesores y alumnos y aprendizaje cooperativo, entre otras. Para los estudiantes, afirma Marqués (2000), las ventajas de las TIC son: “Aprender en menos tiempo, Mejora la eficacia educativa, Mejora de las competencias de expresión y creatividad, Facilidades para trabajar en equipo, mayor proximidad al profesor y apoyo frente a situaciones".

\section{Conclusiones}

A partir de los resultados y discusiones propias de la investigación, la incidencia de los ambientes mediados con TIC para el desarrollo de la lectura inferencial en estudiantes de básica primaria, específicamente grado 3 , de la Institución Educativa José María Berástegui de Ciénaga de Oro Córdoba, y específicamente en lo que tiene que ver con la lectura inferencial, se concluye:

- Que el quehacer pedagógico, desde la aplicabilidad de nuevas estrategias metodológicas, permiten propiciar un clima escolar adecuado, para que los estudiantes permanezcan motivados, tomando la motivación, como el canal que induce a una persona a realizar una acción. Para la docente del grado tercero, fue un espacio de aprendizaje significativo, que contribuyó con sus prácticas de aula, al incorporar y utilizar las herramientas y materiales que no estaban acostumbrados en sus actividades, como videos animados de cuentos, paginas digitales, PC, entre otros, permitiendo de esta manera, a un acercamiento a las herramientas TIC, como medio facilitador para la enseñanza y el fortalecimiento de la competencia semántica comunicativa.

- Se evidenció el mejoramiento en los estudiantes en la competencia semántica comunicativa, específicamente en la lectura inferencial, desarrollando con mayor interés las actividades concernientes con el tema, aumentando su nivel de desempeño de bajo, al nivel medio, reflejando el nivel de interpretar, realizar reflexiones a partir del uso de conocimiento extra-textual, reconociendo el tema de un texto, la intencionalidad del autor y relacionando el texto con los conocimientos sobre géneros o tipos textuales.

- La participación de los estudiantes en el desarrollo de la estrategia didáctica mediada por las TIC, generó en ellos su vinculación activa al proceso de aprendizaje, reconociendo sus fortalezas y debilidades frente a la comprensión lectora, además, el trabajo en el aula permitió el aprendizaje colaborativo, la retroalimentación permanente como parte del proceso de la comprensión de lectura, así como también la a floración de saberes que guardan los estudiantes y que en ocasiones se niegan a emitir por miedo al rechazo o a equivocarse. Lo anterior puede deberse a que las estrategias de 
comprensión lectora planteadas por Solé (1992), permiten que el estudiante construya sus propios significados, mediante la activación de sus conocimientos previos.

- Con el manejo de las mediaciones tecnológicas, el clima de aula mejoró considerablemente, lo que se pudo apreciar en los estudiantes, ya que se mostraban alegres y motivados por aprender de una manera diferente; los docentes pudieron optimizar de manera adecuada el tiempo para la realización de las actividades, y el cooperativismo jugo un papel importante en este proceso. Así mismo, en el aspecto relacionado con la práctica pedagógica, los estudiantes se mostraron más participativos en su gran mayoría, estos se involucraron en las diferentes actividades implementadas, lo cual contribuyó a potencializar su nivel de comprensión lectora sin necesidad de sentir que se les estaba evaluando, sino que el docente realizaba un proceso de evaluación formativa a través del seguimiento, la observación y el uso de rubricas de autoevaluación por parte de los estudiantes.

Por último, se considera que la evaluación a partir de la reflexión permite, a través de la creación de espacios de diálogo, participación y análisis, que estudiantes y docentes logren un verdadero cambio educativo, desde lo cognitivo, tecnológico y lo formativo, identificando aciertos y desaciertos y realizando ajustes durante la misma, que permitan formular y aplicar nuevas acciones sostenibles y potenciadoras de superación personal desde la escuela. Todos los resultados obtenidos, se deben a la motivación que presentaron los estudiantes a través del uso de las nuevas tecnologías. De acuerdo con Duque y Ochoa (2009), es "incuestionable motivación de los estudiantes hacia el uso de las TIC", ya que estos recursos permiten hacer la enseñanza más atractiva y contextualizada; esto permitió que los estudiantes alcanzaran los objetivos de aprendizaje, a partir de clases que para ellos se volvieron más cómodas, atractivas y espontáneas.

\section{Referencias}

Alaís, A., Leguizamón, D., \& Sarmiento, J. (2014). Mejoramiento de la comprensión lectora en estudiantes de cuarto grado de básica primaria mediante el desarrollo de estrategias cognitivas con el apoyo de un recurso tic. (Msc). Universidad de la Sabana, Bogotá D.C. Colombia.

Cassany, D., Luna, M. \& Sanz, G. (2000). Las habilidades lingüísticas. En D. Cassany, M. Luna \& G. Sanz (Ed.), Enseñar lengua (pp. 81-257). Barcelona, España: Graó.

Cassany, D., Luna, M., Sanz, G. (2001). Enseñanza de la Lengua. Barcelona. Graó.

Ceballos, A. (2004). La escuela Tradicional. Universidad Abierta. Material no publicado. Recuperado

http://www.uhu.es/36102/trabajos_alumnos/pt1_11_12/biblioteca/2historia_educacion/esc_ nueva/escuela_tradicional_vs_nueva.pdf [Fecha de Consulta: Abril 28 de 2018].

Cabero, J. (2001). Tecnología Educativa, Diseño y Utilización de medios en la enseñanza. España: Paidos Iberica, S.A.

Charria-Ortiz, V., Sarsosa-Prowesk, K., \& Uribe-Rodríguez, A., \& López-Lesmes, C., \& Arenas-Ortiz, F. (2011). Definición y clasificación teórica de las competencias académicas, profesionales y laborales. Las competencias del psicólogo en Colombia. Psicología desde el Caribe, (28), 133-165. 
Clavijo, J., Maldonado, A., \& Sanjuanelo (2011). Potenciar la comprensión lectora desde la tecnología de la información. Escenarios, 9, 26. (Msc). Universidad Autónoma del Caribe, Barranquilla, Colombia.

Cubas, A. C. (2007). Actitudes hacia la lectura y niveles de comprensión lectora en estudiantes de sexto grado de primaria. (Tesis de Licenciatura) Pontificia Universidad Católica del Perú. Lima, Perú. Recuperado de: http://m.tesis.pucp.edu.pe/repositorio/bitstream/handle/123456789/427/CUBAS_BARRUETO _ANA_ACTITUDES_HACIA_LECTURA.pdf?sequence=1. [Fecha de Consulta: Abril 28 de 2018].

Gardner, H (1994). Estructuras de la mente: La teoría de las inteligencias multiples. Ed Fondo de cultura Economico. Mexico (Pag. 221)

Graesser, A., Bertus, E. \& Magliano, J. (1995). Inference generation during the comprehension of narrative text. En R. Lorch \& E. O'brien (Eds.), Source of Coherence in Reading (pp. 295-320). Hillsdale, New Jersey: Hove.

Hernández Sampieri, Roberto., Carlos Fernández Collado, and Pilar Baptista Lucio. Metodología De La Investigación: Roberto Hernández Sampieri, Carlos Fernández Collado Y Pilar Baptista Lucio. 6a. ed. --. México D.F.: McGraw-Hill, 2014.

Hidalgo, M. (2005). El taller como herramienta pedagógica. En retos y logros, 6(1) pp. 1-84.

Hymes, D. (1971). Competence and performance in linguistic theory. Acquisition of languages: Models and methods. Ed. Huxley and E. Ingram. New York: Academic Press. 3-23.

Hymes, D. (1972). Acerca de la competencia comunicativa (Traducción de Juan Gómez, en Pride y Colmes , Eds, Sociolingüística).

Kemmis, S., \& McTaggart, R. (1988). The action research reader (3rd ed.). Geelong, Australia: Deakin University Press.

Kintsch, W. (1993). Information accretion and reduction in text processing: Inferences. Discourse Processes, 16(1-2), 193-202. doi:10.1080/01638539309544837.

Kisnerman, N. (1977). Los Talleres, ambientes de Formación Profesional. En: El taller, Integración de Teoría y Práctica. De Barros, Nidia A. Gissi, Jorge y otros. Editorial Humanitas. Buenos Aires.

Ministerio de Educación Nacional. (1998). Lineamientos Curriculares en Lenguaje. Bogotá, Colombia: MEN.

Ministerio de Educación Nacional. (2006). Estándares Básicos de Competencias en Lenguaje, Matemáticas, Ciencias y Ciudadanas. Bogotá, Colombia: MEN.

Marqués, P. (2000). Impacto de las tic en educación: funciones y limitaciones. Recuperado de: http://peremarques.pangea.org/siyedu.htm. [Fecha de Consulta: Abril 15 de 2018].

Marqués, P. (2001). Las TIC y sus aportes a la sociedad. Barcelona: UAB.

Parodi, G. (2005). Comprensión de textos escritos. Buenos Aires: Eudeba.

Patton, M. (2002). Qualitative research and evaluation methods. Thousand Oaks: Sage.

Pérez S. (2010). La importancia de las TIC en la escuela. Revista digital para profesionales de la enseñanza. ISSN: 1989-4023. Andalucía: España.

Priestley, M. (1996). Técnicas y estrategias del pensamiento crítico. México: Editorial Trillas.

Quiroz, M. \& Dora P. (2015). Programa de Comprensión Lectora para Niños de Tercer Grado de Educación Primaria de una Institución Educativa Estatal del Distrito de Chorrillos. (Msc). Universidad Ricardo Palma, Lima, Perú.

Sánchez, J. M., Brito, N. (2015). Desarrollo de competencias comunicativas mediante la lectura crítica, escritura creativa y expresión oral. Revista Encuentros, Universidad Autónoma del Caribe, 13 (1), pp. 117-141. 
Solé, I. (1992). Estrategias de lectura (pp. 17-142). Barcelona, España: Editorial Graó.

Taylor, S.J. \& Bogdan R. (1984). Introducción a los métodos cualitativos de investigación. Barcelona, España: Editorial Paidós.

Van Dijk T. (1999). El análisis crítico del discurso. Revista Anthropos, 186, 24, (pp. 23-36). Barcelona.

Villalonga M., Padilla C., \& Burin, D. (2014). Relaciones entre decodificación, conocimiento léxicosemántico e inferencias en niños de escolaridad primaria. Interdisciplinaria, 31(2), 259-274. Recuperado de http://www.scielo.org.ar/scielo.php?script=sci_arttext\&pid=S1668 70272014000200005\&Ing=es\&tIng=es. [Fecha de Consulta: Noviembre 12 de 2017].

Usuga J. (2010). El enfoque semántico comunicativo y la competencia comunicativa. Recuperado de: $\quad$ http://didacticalenguaje.blogspot.com/2010/11/el-enfoque-semantico-comunicativo-yla.html. [Fecha de Consulta: Abril 15 de 2018]. 